\title{
Lebenssituation von jungen Frauen mit UlIrich-Turner-Syndrom nach dem Ende der Wachstumshormontherapie: Ergebnisse einer Umfrage in Deutschland
}

\author{
Life Situation of Young women with Turner Syndrome: \\ Results of a Questionnaire-based Study in Germany
}

Autoren

Helmuth-Günther Dörr ${ }^{1}$, Markus Bettendorf², Gerhard Binder ${ }^{3}$, Jürgen Brämswig ${ }^{4}$, Berthold P. Hauffa ${ }^{5}$, Paul-Martin Holterhus ${ }^{6}$, Klaus Mohnike ${ }^{7}$, Heinrich Schmidt ${ }^{8}$, Günter K. Stalla ${ }^{9}$, Martin Wabitsch ${ }^{10}$, Joachim Wölfle ${ }^{11}$, und die Turner-Syndrom-Vereinigung Deutschland e. V. ${ }^{12}$

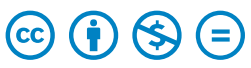

Institute

1 Kinder- und Jugendklinik, Universitätsklinikum Erlangen

2 Sektion Päd. Endokrinologie und Diabetologie, Zentrum

Kinder- und Jugendmedizin, Universitäts-Klinikum Heidelberg

3 Pädiatrische Endokrinologie, Universitätsklinikum Tübingen

4 Pädiatrische Endokrinologie, Universitätsklinikum Münster

5 Kinderklinik II, Pädiatrische Endokrinologie und Diabetologie, Universitätsklinikum Essen

6 Bereich Pädiatrische Endokrinologie und Diabetologie, Klinik für Kinder- und Jugendmedizin I, Universitätsklinikum Schleswig-Holstein, UKSH, Campus Kiel, Universitätsklinikum Schleswig-Holstein, Kiel

7 Universitätskinderklinik, Otto-von-Guericke Universität, Magdeburg

8 Pädiatrische Endokrinologie und Diabetologie, Dr. von Haunersche Kinderklinik der LMU München

9 Medicover Neuroendokrinologie, München

10 Sektion Pädiatrische Endokrinologie und Diabetologie, Universitätsklinik für Kinder- und Jugendmedizin Ulm

11 Universitäts-Kinderklinik, Pädiatrische Endokrinologie und Diabetologie, Bonn

12 Turner-Syndrom-Vereinigung Deutschland e. V.; Geschäftsstelle: Am Bornstück 1, Dornburg

Schlüsselwörter

Fragebogen, Schulabschluss, Gefühlsleben, Sexualität, Fertilität

Key words

questionnaire, emotional life, sexuality, fertility
Bibliografie

DOI https://doi.org/10.1055/a-0841-9918

Online-Publikation: 14.3.2019

Dtsch Med Wochenschr 2019; 144: e87-e93

(c) Georg Thieme Verlag KG, Stuttgart · New York ISSN 0012-0472

Korrespondenzadresse

Professor Dr. med. Helmuth-Günther Dörr

Universitätsklinikum Erlangen

Kinder- und Jugendklinik, Loschgestraße 15, 91054 Erlangen

helmuth-guenther.doerr@uk-erlangen.de

\section{ZUSAMMENFASSUNG}

Einleitung Bei Mädchen und Frauen mit Ullrich-Turner-Syndrom (UTS) werden psychosoziale Auffälligkeiten wie eine ängstliche Persönlichkeit, geringeres Selbstwertgefühl, spätes Lösen vom Elternhaus und/oder späte sexuelle Erfahrungen beschrieben.

Methoden Die Untersuchung wurde 2015 bei 779 Frauen mit UTS im Alter von 25 Jahren (Median) mit einem Fragebogen durchgeführt, der von einer französischen Arbeitsgruppe entwickelt und mit deren Erlaubnis verwendet wurde. Insgesamt konnten 130 Fragebögen (16,7\%) ausgewertet werden. Ergebnisse (MW \pm SD) Nicht verheiratet waren 116 Frauen (89,9\%); 52 Frauen (40\%) lebten im elterlichen Haushalt. Abitur/Fachabitur oder Hochschulabschluss hatten 47,6\%. 60 Frauen (46\%) waren berufstätig, 51 Frauen (39\%) hatten noch keine abgeschlossene Berufsausbildung. Bei $78 \%$ der Frauen wurde die Pubertät im Alter von 14,2 $\pm 2,1$ Jahren eingeleitet. Zum Zeitpunkt der Befragung erhielten $80 \%$ der Frauen eine Hormonersatztherapie. 66 von 93 Frauen (71\%) fanden, dass die Erkrankung das Gefühlsleben negativ beeinflusst, wobei der Bereich „Liebes- und Sexualbeziehungen“ von 44 Frauen $(66,6 \%)$ am häufigsten genannt wurde. Fragen zur Sexualität beantworteten 116 Frauen. Dabei hatten $77 \%$ den ersten Zungenkuss mit 16,4 $\pm 3,6$ Jahren und 62,4\% den ersten Geschlechtsverkehr mit 19,0 \pm 3,4 Jahren. Eine Beziehung zu einem Partner/In über > 6 Monate gaben $81 \%$ der Frauen an (94 Frauen hatten einen männlichen Partner und 
5 Frauen eine Partnerin). Die Frage nach Kinderwunsch wurde von 89 von 124 Frauen bejaht (71,8\%); 38,2\% wollten spontan schwanger werden und $44,9 \%$ hatten eine künstliche Befruchtung oder eine Adoption überlegt.

Diskussion Da die verschiedenen Fragenkomplexe nicht gleich häufig beantwortet wurden, kann spekuliert werden, dass der Stellenwert der Themen nicht gleichwertig war oder dass die Frauen nicht bereit waren, sich mit diesen Fragen auseinanderzusetzen. Die Antworten zeigen, dass neben der ärztlichen Betreuung auch eine psychosoziale Betreuung notwendig ist.

\section{ABSTRACT}

Introduction Psychosocial problems such as anxious personality, low self-esteem, late separation from home and/or late sexual experience have been described in girls and women with Turner syndrome (TS).

Methods The study was performed in 2015 based on a questionnaire that was sent out to 779 women with TS aged 25 years (median). The questionnaire was devised by a French team and used with their permission. In all, 130 questionnaires $(16.7 \%$ ) could be evaluated. The questions from the individual topics were not always completely answered.

Results (mean \pm SD). 116 women (89.9\%) were not married; 52 women (40\%) lived in their parents' home. $47.6 \%$ had a high-school/technical diploma or university degree. 60 women (46\%) had a job; 51 women (39\%) had not completed vocational training. Puberty was induced at the age of $14.2 \pm$ 2.1 years in $78 \%$ of the women. $80 \%$ of the women received hormone replacement therapy at the time of the questionnaire survey. 66 of 93 women (71\%) found that the disease had a negative influence on emotional life. "Love life and sexual relationship" was the topic mentioned most frequently by 44 women (66.6\%). 116 women answered questions on sexuality. Here, $77 \%$ had the first French kiss at the age of $16.4 \pm$ 3.6 years and $62.4 \%$ had sexual intercourse for the first time at the age of $19.0 \pm 3.4$ years. $81 \%$ of the women stated that they had a partner relationship for more than 6 months (94 women had a male partner and 5 had a female partner). The question as to the wish to have children was answered in the affirmative by 89 of 124 women (71.8\%); $38.2 \%$ desired spontaneous pregnancy and $44.9 \%$ had considered in vitro fertilization or adoption.

Discussion The women's answers show that care needs to be improved. There are deficits in the topics of family, emotional life, relationships, sexuality, fertility and pregnancy. Therefore, the medical team should also include psychologists and social workers.

\section{Einleitung}

Das Ullrich-Turner-Syndrom (UTS) ist eine seltene Störung durch partiellen oder vollständigen Verlust eines Geschlechtschromosoms. Die zahlreichen Komorbiditäten erfordern eine multidisziplinäre ärztliche Betreuung [1 - 3]. Bei der Mehrzahl der Frauen mit UTS finden sich auch Defizite in der nonverbalen Kommunikation, der visuell-räumlichen Verarbeitung, der sozial-kognitiven Wahrnehmung und im problemlösenden Denken sowie der Motorik [4-6]. Das Risiko, eine unreife oder ängstliche Persönlichkeit zu entwickeln bzw. sozial isoliert zu leben, ist erhöht [4, 7]. Eine Zusammenstellung der verschiedenen neurokognitiven, akademischen, sozialen und psychologischen Phänotypen des UTS findet sich in der aktuellen Leitlinie [8]. Es wird daher gefordert, dass dem ärztlichen Team auch Psychologen und Sozialpädagogen zugeordnet werden sollten, da die psychologischen Aspekte der Erkrankung oft in den Hintergrund treten.

Der Zugewinn an Körperhöhe nach einer Therapie mit Wachstumshormon $(\mathrm{GH})$ wird positiv beurteilt [9]. Er soll zu einer geringeren psychosozialen Belastung und damit auch zu einer besseren Lebensqualität der betroffenen Frauen führen [10].

Ziel der vorliegenden Untersuchung war es, junge Frauen mit UTS in Deutschland nach dem Ende der Wachstumshormontherapie mittels eines Fragebogens zu verschiedenen Aspekten ihrer aktuellen Lebenssituation zu befragen.

\section{Patienten}

Die vorliegende Studie wurde 2015 durchgeführt. Die Frauen waren zum Zeitpunkt der Datenerhebung im Mittel 24,0 4,2 (SD) Jahre alt. Voraussetzung für die Teilnahme an der Studie war ein Alter $\geq 18$ Jahre und eine Therapie mit Wachstumshormon $(\mathrm{GH})$ im Kindes- und Jugendalter. Für die Erfassung der Daten wurde der Fragebogen verwendet, der bereits 2001 in einer Studie in Frankreich eingesetzt wurde [11]. Der Papierfragebogen wurde auf Deutsch übersetzt und hatte einen Umfang von 11 Seiten. Die Fragenkomplexe betrafen Fragen zur familiären, schulischen und beruflichen Situation, zur medizinischen Betreuung und zur Behandlung mit Wachstumshormon. Ein weiterer Fragenkomplex beinhaltete verschiedene Fragen zu dem Gefühls- und Sexualleben $[10-12]$.

An der Studie beteiligten sich in Deutschland 11 Zentren (10 mit Pädiatrischer Endokrinologie) und die Turner-SyndromVereinigung Deutschland e. V. Insgesamt wurden von den Zentren 779 Fragebögen mit einem Informationsschreiben und vorfrankierten und voradressierten Umschlag (Professor Dörr, Erlangen) an die Frauen verschickt, die im jeweiligen Zentrum über Jahre betreut wurden. Von den Fragebögen kamen 176 $(22,6 \%)$ zurück und 130 Fragebögen (16,7 \%) konnten ausgewertet werden. Die Fragenkomplexe wurden von den Frauen nicht immer vollständig beantwortet.

Die Untersuchung wurde von der Ethikkommission der Medizinischen Fakultät der Friedrich-Alexander-Universität ErlangenNürnberg (Antrag-Nr. 80-13 B) genehmigt. Die Daten wurden anonymisiert erhoben. Die Patientinnen wurden nur nach dem 
Alter, der Postleitzahl des Wohnortes und nach der Mitgliedschaft in der Turner-Syndrom-Vereinigung Deutschland e. V. gefragt. Als Referenzkohorte wurden die Daten zur Jugendsexualität der Bundeszentrale für gesundheitliche Aufklärung (BzGA) aus dem Jahr 2015 [13] und statistische Zahlen, aufbereitet von der Firma Statista $\mathrm{GmbH}$, verwendet [14].

Die statistische Auswertung erfolgte durch die Fa. Anfomed in Möhrendorf. Die Auswertung der Fragebögen erfolgte deskriptiv und die Ergebnisse wurden als prozentualer Anteil/Häufigkeiten und, wenn anwendbar, als Mittelwert und Standardabweichung dargestellt. Statistische Vergleichsberechnungen mit Ermittlung von Wahrscheinlichkeiten wurden nicht durchgeführt, da es sich um eine explorative Vergleichsstudie zur Hypothesengenerierung handelt.

\section{Ergebnisse}

\section{Familie, Schule und Beruf ( $\triangleright$ Tab. 1)}

Zum Zeitpunkt der Befragung im Alter von 24,0 \pm 4,23 Jahren waren 116 Frauen $(89,9 \%)$ nicht verheiratet. 7 Frauen waren verheiratet (5,43\%); eine Patientin war geschieden. Im Haushalt der Eltern wohnten 42 Frauen (32,6\%); 10 Frauen (7,75\%) wohnten bei der Mutter. Mit einem Partner/Partnerin wohnten 28 Frauen (21,7\%), 23 Frauen (17,8\%) wohnten alleine und 17 Frauen $(13,2 \%)$ wohnten in einer Wohngemeinschaft.

Der Anteil der Frauen mit Abitur/Fachabitur lag bei $32 \%$; einen Hochschulabschluss hatten 21 Frauen (16\%). Einen Realschulabschluss (Mittlere Reife) hatten 29\% und einen Hauptschulabschluss $11 \%$ der Frauen. 7 Frauen hatten eine Förderschule besucht und 6 Frauen hatten noch keinen Schulabschluss. Zum Zeitpunkt der Befragung hatten 51 Frauen (39\%) noch keine abgeschlossene Berufsausbildung und 60 Frauen (46\%) waren berufstätig.

\section{Pubertät}

Die Fragen zur Pubertät wurden von 125 Frauen beantwortet. Bei 98 Frauen (78\%) wurde sie im Alter von 14,2 $\pm 2,1$ (MW \pm SD) Jahren mit Östrogenen eingeleitet, bei 27 Frauen (21,6\%) erfolgte der Pubertätsbeginn spontan. Das Alter bei Menarche lag unter einer kombinierten Östrogen/Gestagen-Therapie bei 14,9 \pm 1,6 Jahren. Bei den 27 Mädchen mit spontaner Pubertät erfolgte die Menarche im Alter von 13,9 \pm 1,5 Jahren.

\section{Gefühlsleben}

Die Fragen, ob die Erkrankung „Einfluss auf das Gefühlsleben“ nimmt und wenn ja, in welchem Bereich, wurde von 93 der 130 Frauen (71,5\%) beantwortet. Dabei fanden 66 von 93 Frauen (71\%), dass die Erkrankung das Gefühlsleben negativ beeinflusst, wobei der Bereich „Liebes- und Sexualbeziehungen“ von 44 Frauen $(66,6 \%)$ am häufigsten genannt wurde. Danach folgten die Bereiche „freundschaftliche Beziehungen“ (36,2\%), „berufliches Umfeld“ (26,1\%) und „innerfamiliäres Umfeld“ mit 10,1\%.
- Tab. 1 Daten zur aktuellen Lebenssituation von 130 Frauen mit Ullrich-Turner-Syndrom; beantwortete Fragen n (\%).

\begin{tabular}{|c|c|}
\hline Familie & $n=130$ \\
\hline - ledig & $116(89,2 \%)$ \\
\hline - verheiratet & $14(10,8 \%)$ \\
\hline Wohnsituation & $n=130$ \\
\hline - Eltern & $52(40,0 \%)$ \\
\hline - Partner/Partnerin & $28(21,7 \%)$ \\
\hline - allein & $23(17,8 \%)$ \\
\hline - Wohngemeinschaft & $17(13,2 \%)$ \\
\hline Höchster Schulabschluss & $\mathrm{n}=130$ \\
\hline - Hochschule/Abitur/Fachabitur & $47,6 \%$ \\
\hline - Mittlere Reife & $29,2 \%$ \\
\hline - Hauptschule & $10,8 \%$ \\
\hline - Förderschule & $5,4 \%$ \\
\hline Gefühlsleben & $n=93$ \\
\hline wird negativ beeinflusst & $66 / 93(71 \%)$ \\
\hline - Bereich Liebesbeziehungen/Sexualität & $66 \%$ \\
\hline - Bereich Freundschaften & $36 \%$ \\
\hline Pubertät & $n=125$ \\
\hline 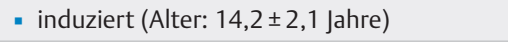 & $98(78,4 \%)$ \\
\hline - spontan & $27(21,6 \%)$ \\
\hline Sexualität & $n=116$ \\
\hline - Alter erster Zungenkuss (16,4 × 3,6 Jahre) & $90(77,5 \%)$ \\
\hline $\begin{array}{l}\text { - Alter erster Geschlechtsverkehr } \\
\text { (19,0 } 0 \text { 3,4 Jahre) }\end{array}$ & $73(62,9 \%)$ \\
\hline - Beziehung zu einem Partner/In >6 Monate & $75 / 92(81,5 \%)$ \\
\hline Kinderwunsch/Schwangerschaft & $n=124$ \\
\hline ja & $89(71,8 \%)$ \\
\hline - spontan & $34(38,2 \%)$ \\
\hline - In-vitro-Fertilisation/Adoption & $40(44,9 \%)$ \\
\hline
\end{tabular}

\section{Sexualität}

Die Fragen zum ersten Kuss und Geschlechtsverkehr wurde von 116 Frauen beantwortet. 90 Frauen (77\%) hatten erste Erfahrungen mit einem Zungenkuss im Alter von 16,4 \pm 3,6 (MW \pm SD) Jahren (Median: 16 Jahre). Den ersten Geschlechtsverkehr hatten 73 Frauen (62,9\%) im Alter von 19,0 \pm 3,4 Jahren (Median: 18 Jahre). Die Frage nach der Dauer der Beziehung zu einem Partner, die mehr als 6 Monate dauerte, wurde von 75 von 92 Frauen (81\%) bejaht. Die Frage nach dem Geschlecht des Partners wurde von 99 Frauen beantwortet. Davon hatten 94 Frauen einen Partner und 5 Frauen eine Partnerin.

\section{Kinderwunsch/Schwangerschaft}

Die Frage wurde von 124 Frauen beantwortet. Insgesamt hatten 89 Frauen (71,8\%) einen Kinderwunsch, 12 Frauen keinen (9,7\%) 
und 23 Frauen (18,5\%) hatten dazu noch keine Meinung. Von den 89 Frauen mit Kinderwunsch wollten 34 Frauen (38,2\%) spontan schwanger werden, während von 40 Frauen (44,9\%) die Möglichkeit der künstlichen Befruchtung oder eine Adoption in Erwägung gezogen wurden. 5 Frauen waren Mütter (4\%) und hatten ein gesundes Kind geboren; 3 Frauen wurden spontan und 2 Frauen nach einer künstlichen Befruchtung schwanger.

Die Frage nach der Bezugsperson, mit der man über Kinderwunsch und Schwangerschaft sprechen kann, wurde von 120 Frauen (92\%) beantwortet. Als Ansprechpartner (Mehrfachnennungen waren möglich) wurden am häufigsten die Eltern (65\%), gefolgt von Partnern/Freunden/Innen (45\%), dem Hausarzt (25\%) und den Geschwistern (22\%) angegeben.

\section{Diskussion}

Die vorliegende Arbeit analysiert die Lebenssituation von Frauen mit Ullrich-Turner-Syndrom (UTS) in Deutschland nach dem Ende der Wachstumshormontherapie im Kindes- und Jugendalter. Eine vergleichbare Untersuchung wurde bei Frauen mit UTS bisher in Deutschland nicht durchgeführt. Die Befragung wurde schriftlich mittels Papierfragebogen durchgeführt. Diese Datenerfassung gilt in der medizinischen Forschung traditionell als die erste Wahlmöglichkeit. Die anonymisierte Befragung mittels Papierfragebogen gibt den Befragten die Möglichkeit, dass sie völlig frei einzelne Fragenkomplexe beantworten oder auch nicht beantworten können, ohne dass man bei der Bewertung der Daten Rückschlüsse auf die Befragten ziehen kann. Wir gehen davon aus, dass daher auch sensible Fragen, wie z. B. nach dem Alter beim ersten Geschlechtsverkehr, eher beantwortet werden als in einem strukturierten Interview, wo der Befragte entweder telefonisch oder direkt von dem Fragesteller von Angesicht zu Angesicht befragt wird.

Die Ergebnisse können nicht auf die Population aller Frauen mit UTS generalisiert werden, da unsere Studie 2 Voraussetzungen hatte: 1. Therapie mit Wachstumshormon im Kindesalter und 2. Alter der Frauen. Es gibt in Deutschland kein Register, das die Daten von Frauen mit Turner-Syndrom erfasst. In der TurnerSyndrom-Vereinigung Deutschland e. V. sind nur ca. 3-5\% der betroffenen Frauen registriert.

In der vorliegenden Untersuchung wurde der gleiche Papierfragebogen wie in der französischen Studie verwendet. In Frankreich wurde die Studie 2001 durchgeführt und die Ergebnisse wurden zwischen 2005 und 2009 publiziert [10 - 12]. Unsere Ergebnisse können aber nur zum Teil mit denen aus Frankreich verglichen werden ( $\triangleright$ Tab. 2), da sich in den publizierten Arbeiten keine Daten finden. Die Anzahl der angeschriebenen Frauen war in beiden Ländern ähnlich hoch (Frankreich $\mathrm{n}=891$ und Deutschland $n=779$ ), aber die Rücklaufquote der Befragung lag in Frankreich bei $69 \%$ und in Deutschland bei 22,6\%. Das mittlere Alter der Frauen mit UTS in der vorliegenden Studie war mit 24 Jahren (Altersbereich 18-35 Jahre) nahezu gleich mit dem Alter der Frauen in der französischen Studie von Carel et al. mit 22,6 Jahren (Bereich: 18,3-31,2 Jahre) [10, 12].

Aufgrund des Alters ist es nicht verwunderlich, dass die meisten Frauen $(89,9 \%)$ noch nicht verheiratet waren und dass $40 \%$ der Frauen mit UTS im elterlichen Haushalt leben. Auf der anderen
Seite erscheint die Zahl von $40 \%$ aber relativ hoch, wenn man die Zahl in Relation zu den Angaben bei 25-jährigen Frauen in Deutschland setzt, von denen nur ca. $20 \%$ bei den Eltern leben [14]. Daher würde die Zahl von $40 \%$ eher die bekannte Annahme bestätigen, dass sich Frauen mit UTS eher spät vom Elternhaus lösen [15].

Der Anteil der Frauen mit UTS in der vorliegenden Studie mit Abitur/Fachabitur (32\%) und Mittlerer Reife (29\%) entspricht den aktuellen Zahlen zum höchstem Schulabschluss der Frauen in Deutschland (Abitur/Fachabitur 28,2\%; Mittlere Reife 28,5\%) [14]. Eine Untersuchung aus den USA konnte zeigen, dass Frauen mit UTS oft sogar einen höheren Schulabschluss haben als die jeweilige Referenzpopulation [16].

Die Angaben zur Pubertätsinduktion waren in Deutschland und Frankreich gleich. Die Pubertät wurde bei $78 \%$ der deutschen Mädchen mit durchschnittlich 14,2 Jahren und in Frankreich bei 72 \% der Mädchen mit 14,9 Jahren induziert. Nach den Daten der deutschen KiGGS-Studie zur spontanen Pubertätsentwicklung bei Jugendlichen haben $50 \%$ der gesunden Mädchen im Mittel mit 10,9 Jahren bereits das Tanner-Stadium B2 der Brustentwicklung erreicht [17]. Dies bedeutet, dass die Pubertät bei den Mädchen mit UTS eher spät induziert wurde. Das Tempo der Pubertät beeinflusst bei gesunden Mädchen den Beginn der Sexualität $[18,19]$. Es ist daher nicht verwunderlich, dass Frauen mit UTS im Vergleich zu ihren Altersgenossinnen sexuelle Erfahrungen später machen [12, 20]. Die sexuellen Erfahrungen der Frauen mit UTS wurden mittels mehrerer Fragen beurteilt und von 116 Frauen beantwortet. So wurde danach gefragt, ob sie Erfahrungen mit Küssen oder Treffen (Dates) hatten und ob sie Geschlechtsverkehr (Ja/Nein und das Alter beim ersten Mal) gehabt hatten. In unserer Kohorte bejahten 77 \% der Frauen die Frage nach einem in der Vergangenheit stattgefundenen Zungenkuss, während in der Studie zur Jugendsexualität der Bundeszentrale für gesundheitliche Aufklärung (BzGA) die Frage von $98 \%$ der Mädchen im gleichen Altersbereich bejaht wurde [21].

Der erste Geschlechtsverkehr wurde von $63 \%$ der Frauen mit UTS in unserer Studie mit durchschnittlich 19 Jahren berichtet; $37 \%$ hatten noch keinen Geschlechtsverkehr. Diese Zahl lag deutlich höher als in der französischen Studie, wo 23 \% der Frauen mit UTS den ersten Geschlechtsverkehr mit 20 Jahren berichteten [12]. In einer Studie aus USA mit 80 betroffenen Frauen (mittleres Alter: 34 Jahre) hatten 55 \% der Frauen im Mittel mit 23 Jahren Geschlechtsverkehr [22]. Im Vergleich dazu haben in der BzGA-Studie $94 \%$ der gesunden Frauen in der Altersgruppe der 24-Jährigen den ersten Geschlechtsverkehr [21] und in Frankreich liegt der Anteil der gesunden Frauen vor dem 20. Lebensjahr bei 85 \% [12].

Die betroffenen deutschen Frauen waren in der vorliegenden Studie im Vergleich zu den UTS-Frauen in den anderen Untersuchungen früher sexuell aktiv. Im Vergleich mit den Frauen der Referenzpopulation waren sie aber in dem jeweiligen Altersbereich später sexuell aktiv [15]. Dabei können Faktoren wie Schüchternheit und Ängstlichkeit in Bezug auf Sozialkontakte sowie ein niedrigeres Selbstwertgefühl im Vergleich zu gesunden Frauen auch eine Rolle spielen [23]. Die französische Studie konnte auch zeigen, dass das Alter beim ersten Kuss der Mädchen mit UTS positiv mit dem spontanen Auftreten der Pubertät und negativ mit assoziierten kardialen Problemen korreliert [12]. Die aktuelle Leit- 
- Tab. 2 Vergleich von Daten zur Lebenssituation von jungen Frauen mit Ullrich-Turner-Syndrom in Deutschland und Frankreich. Die Ergebnisse aus Frankreich wurden aus verschiedenen Publikationen zusammengestellt [10-12].

\begin{tabular}{|c|c|c|}
\hline & Deutschland & Frankreich \\
\hline Patientenidentifikation & Kliniken + Selbsthilfegruppe & Nationales Register \\
\hline Design der Studie & Papierfragebogen & Papierfragebogen \\
\hline Jahr der Untersuchung & 2015 & 2001 \\
\hline Rücklauf der Fragebogen, N (\%) & $176(22,6 \%)$ & $568(63,7 \%)$ \\
\hline Auswertbare Fragebogen, N (\%) & $130(16,7 \%)$ & keine Angaben \\
\hline Alter der Frauen (MW $\pm S D)$ & $24,0 \pm 4,2$ Jahre & $22,6 \pm 2,6$ Jahre \\
\hline Altersbereich (Jahre) & $18,0-35,0$ & $18,3-31,2$ \\
\hline Karyotyp (45 X) & keine Daten & $53 \%$ \\
\hline \multicolumn{3}{|l|}{ Schulabschluss } \\
\hline - Hochschule & $16,1 \%$ & \multirow{2}{*}{$\begin{array}{l}\text { zusammen } \\
39 \%\end{array}$} \\
\hline - Abitur/Fachabitur & $31,5 \%$ & \\
\hline - mittlere Reife & $29,2 \%$ & keine Angaben \\
\hline - Hauptschule & $10,8 \%$ & $25 \%$ \\
\hline - Förderschule & $5,4 \%$ & keine Angaben \\
\hline \multicolumn{3}{|l|}{ Beruf } \\
\hline - Alter <20 Jahre, N (\%) & $23(17,7 \%)$ & $203(36 \%)$ \\
\hline - berufstätig (Vollzeit), N (\%) & $52(40 \%)$ & $163(29 \%)$ \\
\hline \multicolumn{3}{|l|}{ Pubertät } \\
\hline - induziert, N (\%) & $98(78 \%)$ & $405(72 \%)$ \\
\hline " spontan, N (\%) & $27(22 \%)$ & $127(21 \%)$ \\
\hline - Alter (Jahre) bei induz. Pubertät & $14,2 \pm 2,1$ Jahre & $14,9 \pm 1,9$ Jahre \\
\hline \multicolumn{3}{|l|}{ Beziehungen (Liebe, Sexualität) } \\
\hline - Erfahrungen mit Küssen und Verabredungen (Dates), N (\%) & $90(77 \%)$ & $165(29 \%)$ \\
\hline - Geschlechtsverkehr mit 20 Jahren & $51(44 \%)$ & $132(23 \%)$ \\
\hline - keinen Geschlechtsverkehr & $44(37 \%)$ & $187(33 \%)$ \\
\hline
\end{tabular}

linie empfiehlt den Beginn der Pubertätsinduktion bei den betroffenen Mädchen früh zwischen 11 und 12 Jahren [8].

Frauen mit UTS haben eine normale weibliche Geschlechtsidentität, haben in der Regel aber weniger Sexualkontakte und Sozialkontakte als eine Referenzpopulation von erwachsenen Frauen [7, 22, 24]. Die Mehrzahl der betroffenen Frauen fand, dass die Erkrankung das Gefühlsleben beeinflusst. Die diskrepanten Zahlen zur Häufigkeit der beantworteten Fragen zur Sexualität, Gefühlsleben oder Beziehungen zeigen, dass diese Themen bei den Frauen einen unterschiedlichen Stellenwert haben. Man könnte aber auch spekulieren, dass die betroffenen Frauen nicht bereit waren, diese Fragen zu beantworten oder sich mit diesen Fragen auseinanderzusetzen.

Die Mehrzahl der Frauen mit UTS ist aufgrund der Gonadendysgenesie unfruchtbar $[25,26]$. Die bestehende Infertilität wird von den Betroffenen als größte Einschränkung empfunden [27]. Spontane Schwangerschaften sind wie in unserer Studie mit ca. $5 \%$ sehr selten $[28,29]$, zudem ist die Rate an Fehlgeburten nach spontaner Schwangerschaft erhöht [8]. Von 8095 der gesunden
Frauen wird im Altersbereich der 14- bis 25-Jährigen der Kinderwunsch grundsätzlich bejaht [13]. Die Frage nach einem Kinderwunsch wurde in unserer Kohorte von $72 \%$ der Frauen bejaht. Dabei wollten $38 \%$ der Frauen spontan schwanger werden und 44,9\% hatten die Möglichkeit einer künstlichen Befruchtung bzw. einer Adoption erwogen. Wenn man aber berücksichtigt, dass ca. $80 \%$ der Frauen in unserer Kohorte eine hormonelle Ersatztherapie bekamen, dann kann man die Einschätzung der Frauen zur Fertilität und zu einer spontanen Schwangerschaft nicht teilen. Daher sollte die Beratung der betroffenen Frauen über die Fertilität dringend verbessert [30 - 33] und auch die maternalen und fetalen Risiken einer Schwangerschaft ausführlich diskutiert werden [34].

Die Defizite in der Kommunikation dieser angesprochenen Themenfelder liegen auch im familiären Umfeld. Laut BzGA nimmt die Kommunikation zwischen Eltern und Kindern über sexuelle Themen mit steigendem Alter zu. Aus der Altersgruppe der 21- bis 25-Jährigen bejahen $79 \%$ der jungen Frauen deutscher Herkunft Gespräche über Sexualität und Partnerschaft [21]. In der 
vorliegenden Untersuchung wurden die Eltern von $65 \%$ der Frauen mit UTS als die wichtigsten Ansprechpartner benannt. In einer Studie aus den USA hatten aber von 215 Eltern mit einem Kind mit UTS nur 50 \% der Eltern mit den Mädchen über Themen wie Sexualität und Fertilität gesprochen [35]. Die Probleme werden auch bei Familien mit chronisch kranken Kindern beschrieben und als mögliche Hindernisse in der Kommunikation wurden Faktoren wie mangelndes Wissen, Schamgefühl oder der Wunsch, das Kind zu beschützen, identifiziert.

Die Daten lassen keine Rückschlüsse auf die Lebensqualität der Frauen zu. In der französischen Studie erhielten die Frauen auch zusätzliche psychometrische Fragebogen zur Erfassung der Lebensqualität [11]. Dabei konnte gezeigt werden, dass die Körperhöhe nach dem Ende der GH-Therapie keinen Einfluss auf die Lebensqualität der Frauen hatte [7, 10], während zu hohe Therapieerwartungen mit einer niedrigen Lebensqualität verbunden waren [10]. Eine späte Pubertätsinduktion nach dem Alter von 15 Jahren hatte ebenfalls einen negativen Einfluss auf die Lebensqualität [10]. Als weitere Faktoren mit einem negativen Einfluss auf die Lebensqualität der betroffenen Frauen wurden Hörstörungen, das Lebensalter bei Diagnosestellung, d. h. je später die Diagnosestellung desto schlechter, und schulische Probleme gefunden [36].

\section{Zusammenfassung}

Die Ergebnisse müssen kritisch bewertet werden. Ein Bias kann nicht ausgeschlossen werden, da die Rücklaufquote der Fragebögen bei 23 \% lag. Für die Analyse standen 130 Fragebögen (16,7\%) zur Verfügung und einzelne Fragenkomplexe wurden nicht gleich häufig beantwortet. Die untersuchte Kohorte ist nicht repräsentativ für die Frauen mit UTS in Deutschland. Von den Frauen in unserer Studie wurden Fragen zur Sexualität von $89 \%$, Fragen zum Gefühlsleben von $71 \%$ und Fragen zu Beziehungen von $53 \%$ beantwortet. Die diskrepanten Zahlen zur Häufigkeit der beantworteten Fragen zeigen, dass die Themen bei den Frauen einen unterschiedlichen Stellenwert haben. Es könnte aber auch sein, dass die Frauen nicht bereit waren, sich mit diesen Fragen auseinanderzusetzen. Sexuelle Erfahrungen werden bei den betroffenen Frauen etwas später und seltener gemacht werden als in der Normalpopulation. Die Antworten zu den Themen Fertilität und Schwangerschaft zeigen, dass dringend eine bessere Aufklärung und Kommunikation aller Beteiligten notwendig sind.

\section{KERNAUSSAGEN}

- Die Kohorte ist nicht repräsentativ für Frauen mit UTS in Deutschland.

- Der Anteil der Frauen mit Abitur/Fachabitur und Mittlere Reife entspricht den aktuellen Zahlen zum höchsten Schulabschluss von Frauen in Deutschland.

- Die betroffenen Frauen waren im Vergleich mit den UTSFrauen in anderen Untersuchungen früher sexuell aktiv, aber später im Vergleich mit Frauen der Referenzpopulation.

- Die Frage nach einem Kinderwunsch wurde von 72 \% der Frauen bejaht.
- Die Mehrzahl der Frauen fand, dass die Erkrankung das Gefühlsleben negativ beeinflusst.

- Die Daten lassen keine Rückschlüsse auf die Lebensqualität der Frauen zu.

Interessenkonflikt

Die Autoren geben an, dass kein Interessenkonflikt besteht. Ein Teil der Daten wurde im Rahmen einer medizinischen Doktorarbeit verwendet. Wir danken der Firma Novo Nordisk Pharma $\mathrm{GmbH}$, die die Kosten der Studie (Übersetzung des Fragebogens, Druckkosten, Briefumschläge, Portokosten) durch eine Spende finanziell unterstützt hat.

\section{Literatur}

[1] Ranke MB, Pfluger $\mathrm{H}$, Rosendahl W et al. Turner syndrome: spontaneous growth in 150 cases and review of the literature. Eur J Pediatr 1983; 141: $81-88$

[2] Lyon A], Preece MA, Grant DB. Growth curve for girls with Turner syndrome. ArchDisChild 1985; 60: 932 -935

[3] Ranke MB, Saenger P. Turner's syndrome. Lancet 2001; 358: 309-314

[4] McCauley E, Ross JL, Kushner $\mathrm{H}$ et al. Self-esteem and behavior in girls with Turner syndrome. J Dev Behav Pediatr 1995; 16: 82-88

[5] McCauley E, Kay T, Ito J et al. The Turner syndrome: cognitive deficits, affective discrimination, and behavior problems. Child development 1987; 58: $464-473$

[6] Sybert VP, McCauley E. Turner's syndrome. N Engl J Med 2004; 351: $1227-1238$

[7] Amundson E, Boman UW, Barrenas ML et al. Impact of growth hormone therapy on quality of life in adults with turner syndrome. J Clin Endocrinol Metab 2010; 95: 1355-1359

[8] Gravholt CH, Andersen NH, Conway GS et al. Clinical practice guidelines for the care of girls and women with Turner syndrome: proceedings from the 2016 Cincinnati International Turner Syndrome Meeting. Eur J Endocrinol 2017; 177: G1 - G70

[9] Baxter L, Bryant J, Cave CB et al. Recombinant growth hormone for children and adolescents with Turner syndrome. Cochrane database of systematic reviews (Online) 2007: CD003887. doi:10.1002/14651858. CD003887.pub2

[10] Carel JC, Ecosse E, Bastie-Sigeac I et al. Quality of life determinants in young women with turner's syndrome after growth hormone treatment: results of the StaTur population-based cohort study. J Clin Endocrinol Metab 2005; 90: $1992-1997$

[11] Devernay M, Ecosse E, Coste J et al. Determinants of medical care for young women with Turner syndrome. J Clin Endocrinol Metab 2009; 94: $3408-3413$

[12] Carel JC, Elie C, Ecosse E et al. Self-esteem and social adjustment in young women with Turner syndrome-influence of pubertal management and sexuality: population-based cohort study. J Clin Endocrinol Metab 2006; 91: 2972-2979

[13] Bode H, Heßling A. Jugendsexualität 2015. Die Perspektive der 14-bis 25-Jährigen. Ergebnisse einer aktuellen Repräsentativen Wiederholungsbefragung. In. Köln: Bundeszentrale für gesundheitliche Aufklärung. 2015

[14] Statista GmbH. Frauen in Deutschland 2017. Im Internet: https:// destatistacom/themen/1775/frauen-in-deutschland; Stand: August 2018

[15] Naess EE, Bahr D, Gravholt CH. Health status in women with Turner syndrome: a questionnaire study on health status, education, work 
participation and aspects of sexual functioning. Clin Endocrinol (Oxf) 2010; 72: $678-684$

[16] Gould HN, Bakalov VK, Tankersley C et al. High levels of education and employment among women with Turner syndrome. Journal of women's health (2002) 2013; 22: 230-235

[17] Kahl H, Schaffrath RA, Schlaud M. Sexual maturation of children and adolescents in Germany. Results of the German Health Interview and Examination Survey for Children and Adolescents (KiGGS). BundesgesundheitsblattGesundheitsforschungGesundheitsschutz 2007; 50 : $677-685$

[18] Goodson P, Evans A, Edmundson E. Female adolescents and onset of sexual intercourse: a theory-based review of research from 1984 to 1994. J Adolesc Health 1997; 21: $147-156$

[19] Lam TH, Shi H], Ho LM et al. Timing of pubertal maturation and heterosexual behavior among Hong Kong Chinese adolescents. Archives of Sexual Behavior 2002; 31: 359-366

[20] Ehrhardt AA, Greenberg N, Money ]. Female gender identity and absence of fetal gonadal hormones: Turner's syndrome. Johns Hopkins Med J 1970; 126: $237-248$

[21] Bode H, Hessling A. Jugendsexualität 2015. Die Perspektive der 14- bis 25-Jährigen. Ergebnisse einer aktuellen repräsentativen Wiederholungsbefragung. Köln: Bundeszentrale für gesundheitliche Aufklärung. 2015

[22] Pavlidis K, McCauley E, Sybert VP. Psychosocial and sexual functioning in women with Turner syndrome. Clin Genet 1995; 47: 85-89

[23] Schmidt PJ, Cardoso GM, Ross JL et al. Shyness, social anxiety, and impaired self-esteem in Turner syndrome and premature ovarian failure. JAMA 2006; 295: 1374-1376

[24] Fjermestad KW, Naess EE, Bahr D et al. A 6-year Follow-up survey of health status in middle-aged women with Turner syndrome. Clin Endocrinol (Oxf) 2016; 85: 423-429

[25] Hewitt JK, Jayasinghe Y, Amor DJ et al. Fertility in Turner syndrome. Clin Endocrinol (Oxf) 2013; 79: 606-614
[26] Bouet PE, Godbout A, El Hachem $\mathrm{H}$ et al. Fertility and Pregnancy in Turner Syndrome. Journal of obstetrics and gynaecology Canada: JOGC = Journal d'obstetrique et gynecologie du Canada 2016; 38: 712-718

[27] Sutton E], Mclnerney-Leo A, Bondy CA et al. Turner syndrome: four challenges across the lifespan. Am J Med Genet A 2005; 139A: 57 - 66

[28] Bryman I, Sylven L, Berntorp K et al. Pregnancy rate and outcome in Swedish women with Turner syndrome. Fertil Steril 2011; 95: 2507 2510

[29] Bernard V, Donadille B, Zenaty D et al. Spontaneous fertility and pregnancy outcomes amongst 480 women with Turner syndrome. Hum Reprod 2016; 31: $782-788$

[30] Oktay K, Bedoschi G, Berkowitz K et al. Fertility Preservation in Women with Turner Syndrome: A Comprehensive Review and Practical Guidelines. Journal of Pediatric and Adolescent Gynecology 2016; 29: 409-416

[31] Sutton EJ, Young J, Inerney-Leo A et al. Truth-telling and Turner Syndrome: the importance of diagnostic disclosure. J Pediatr 2006; 148 : $102-107$

[32] Borgstrom B, Hreinsson J, Rasmussen C et al. Fertility preservation in girls with turner syndrome: prognostic signs of the presence of ovarian follicles. J Clin Endocrinol Metab 2009; 94: 74-80

[33] Grynberg M, Bidet M, Benard J et al. Fertility preservation in Turner syndrome. Fertil Steril 2016; 105: 13-19

[34] Schulze C, Heusinger K, Dörr HG et al. Das Ullrich-Turner-Syndrom aus gynäkologischer und geburtshilflicher Sicht. Frauenheilkunde up2date 2013; 7: 16

[35] King JE, Plamondon J, Counts D et al. Barriers in communication and available resources to facilitate conversation about infertility with girls diagnosed with Turner syndrome. J Pediatr Endocrinol Metab 2016; 29 : $185-191$

[36] Boman UW, Bryman I, Moller A. Psychological well-being in women with Turner syndrome: somatic and social correlates. J Psychosom Obstet Gynaecol 2004; 25: $211-219$ 